\title{
Tiling the Line with Triples
}

\author{
Aaron Meyerowitz \\ Florida Atlantic University, Boca Raton FL 33432
}

received March 5, 2001, revised May 10, 2001, accepted May 22, 2001.

\begin{abstract}
It is known the one dimensional prototile $\{0, a, a+b\}$ and its reflection $\{0, b, a+b\}$ always tile some interval. The subject has not received a great deal of further attention, although many interesting questions exist. All the information about tilings can be encoded in a finite digraph $D_{a b}$. We present several results about cycles and other structures in this graph. A number of conjectures and open problems are given.

In [Go] an elegant proof by contradiction shows that a greedy algorithm will produce an interval tiling. We show that the process of converting to a direct proof leads to much stronger results.
\end{abstract}

Keywords: Tiling, one dimension, direct proof

\section{Introduction}

The results of this paper have to do with tiling intervals and other sets in $\mathbb{Z}$ with translates of a 3-element set $\mathcal{A}=\{0, a, a+b\}$ and its reflection $\mathcal{B}=\{0, b, a+b\}$. An elegant proof by contradiction [Go] shows that, for $0<a<b$, the simple greedy rule use $\mathcal{A}$ if possible tiles an interval of length $x_{a b} \leq 2^{a+b-1}$. That upper bound being the number of intermediate states which could potentially come up.

A directed graph $D_{a b}$ with these $2^{a+b-1}$ states as nodes captures all of the information about potential tilings. We conjecture, among many other things, that all its cycles belong to one connected component in the case $\operatorname{gcd}(a, b)=1$ and $\{0, a, a+b\} \neq\{0,1,2\} \bmod 3$. In this case it appears that every interval (or other periodic) tiling uses as many copies of $\mathcal{A}$ as $\mathcal{B}$. Essentially, there should be some height function measuring the excess/deficit of $\mathcal{A}$ over $\mathcal{B}$ tiles. In contrast, we prove that there are many components when $\operatorname{gcd}(a, b)>1$ and also when $\{0, a, a+b\}=\{0,1,2\} \bmod 3$.

It is not hard to improve the bound $x_{a b} \leq 2^{a+b-1}$ by a few orders of magnitude. A detailed analysis of the actual behavior [Me] shows that $x_{a b}=a(b+r)$ provided $b=2 q a+r>3 a, \operatorname{gcd}(a, b)=1$ and $-a<r<a$. The greedy rule converts $D_{a b}$ into a discrete dynamic system. A duality relation is given which proves quite useful in probing its structure. The proof by contradiction is easily adjusted to show that the greedy rule, started at any "short" initial state, returns to that state. It is clear that the intermediate states of these cycles include a broader class of states although it is not immediate how to characterize them.

An ideologically motivated attempt to convert to a direct proof leads to a stronger result and the desired characterization. The paper includes a number of conjectures and problems, all open. 


\section{Definitions}

Where convenient, $[0, b)=\{0,1,2, \cdots, b-1\}$. The symbol $\uplus$ denotes disjoint union. Set difference $R \backslash$ $S=\{r \in R \mid r \notin S\}$ is only used in the case that $S \subseteq R$.

For sets $U$ and $V$ and any $x$ define

- translation $U+x=\{u+x \mid u \in U\}$.

- $\operatorname{direct} \operatorname{sum} U \oplus V=\{u+v \mid u \in U$ and $v \in V\}=\biguplus_{v \in V} U+v$

- dilation $x U=\{x u \mid u \in U\}$

- reflection $-U=\{-u \mid u \in U\}$.

In a direct sum $W=U \oplus V$, each $w \in W$ has a unique expression $w=u+v$ with $u \in U$ and $v \in V$. The main uses of dilation are $m \mathbb{Z}$ and the special case of reflection.

Fix integers $0<a<b$, set $n=a+b$, and consider the two prototiles

$$
\mathcal{A}=\{0, a, n\}=-\mathcal{B}+n \text { and } \mathcal{B}=\{0, b, n\}=-\mathcal{A}+n .
$$

To talk about both prototiles at once, let $\mathcal{C}=\{0, c, n\}$ where $c=a$ or $b$ according as $\mathcal{C}=\mathcal{A}$ or $\mathcal{B}$. A tile is any set $\mathcal{A}+e=\{e, a+e, n+e\}$ or $\mathcal{B}+e=\{e, b+e, n+e\}$. A set $U$ is tiled by $\mathcal{A}$ and $\mathcal{B}$ if it is partitioned into disjoint tiles. This partition $\mathcal{A} \oplus P_{\mathcal{A}} \biguplus \mathcal{B} \oplus P_{\mathcal{B}}=U$ is called a $U$-tiling. Then each tile is $T=\mathcal{A}+\min T$ or $T=\mathcal{B}+\min T$. The placement set $P_{\mathcal{A}}$ is the set of positions occupied by the start of an $\mathcal{A}$-tile. We often index the tiles in such a way that $\min T_{i}<\min T_{j}$ for $i<j$. An obvious, yet useful, observation is

Lemma 2.1 Given a set $W=U \oplus V$ and a tiling $\mathcal{A} \oplus P_{\mathcal{A}} \biguplus \mathcal{B} \oplus P_{\mathcal{B}}=U, \mathcal{A} \oplus\left(P_{\mathcal{A}} \oplus V\right) \biguplus \mathcal{B} \oplus\left(P_{\mathcal{B}} \oplus V\right)$ is a tiling of $W$.

We will be interested in the tilings by $\mathcal{A}$ and $\mathcal{B}$, if any, of finite intervals $[0, m), \mathbb{Z}_{m}=\mathbb{Z} / m \mathbb{Z}$, the half line $\mathbb{N}$, and the line $\mathbb{Z}$. A $\mathbb{Z}_{m}$-tiling is a tiling of some system of residues $\bmod m$. Of course, this requires $m=3 k$ for some $k$, as does the special case $[0, m)$. Actually, $\mathcal{A}$ and $\mathcal{B}$ always tile some interval. A harder question is what length intervals can be tiled. A tiling of $\mathbb{N}$ or $\mathbb{Z}$ is periodic with period $p>0$ if $P_{\mathcal{A}}+p \subseteq P_{\mathcal{A}}$ and $P_{\mathcal{B}}+p \subseteq P_{\mathcal{B}}$. Thus each period involves $p / 3$ tiles. A periodic $\mathbb{N}$-tiling is simply a repeated tiling of $[0, p)$.

We will return to the following simple example several times.

Example 1 Consider the prototiles $\mathcal{A}=\{0,2,5\}$ and $\mathcal{B}=\{0,3,5\}$. The tiles

$T_{0}=\mathcal{A}+0, T_{1}=\mathcal{A}+1, T_{2}=\mathcal{B}+4, T_{3}=\mathcal{A}+8, T_{4}=\mathcal{B}+11, T_{5}=\mathcal{B}+12$ constitute a tiling of the interval

$$
[0,18)=\{0,1,2, \cdots 17\}=\mathcal{A} \oplus\{0,1,8\} \biguplus \mathcal{B} \oplus\{4,11,12\} .
$$

Since $[0,18)+18 \mathbb{N}=\mathbb{N}$, Lemma 2.1 notes that the same 6 step sequence could be repeated to tile $\mathbb{N}$ with period 18. Likewise,

$$
\mathcal{A} \oplus(\{0,1,8\}+18 \mathbb{Z}) \biguplus \mathcal{B} \oplus(\{4,11,12\}+18 \mathbb{Z})=\mathbb{Z} .
$$


We might illustrate the successive situations of this $\mathbb{Z}$-tiling with diagrams

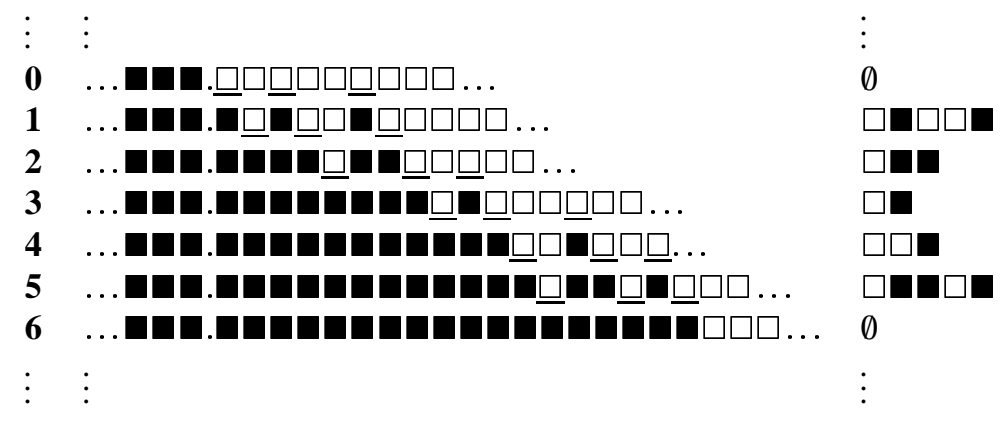

The decimal point is between position -1 and position 0 . Each state diagram on the right determines the corresponding situation, up to translation.

Using the same tiles,

$$
\mathcal{A} \biguplus(\mathcal{B}+4)=\mathcal{A} \oplus\{0\} \biguplus \mathcal{B} \oplus\{4\}=\{0,2,5,4,7,9\}
$$

is a system of residues mod 6 ; a tiling of $\mathbb{Z}_{6}$. Hence $\mathcal{A} \oplus 6 \mathbb{Z} \biguplus \mathcal{B} \oplus(6 \mathbb{Z}+4)=\mathbb{Z}$

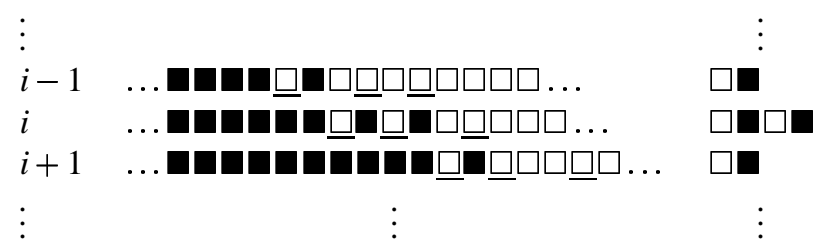

A duality, introduced more formally in definition 3, can be seen in these tables. Visually, white denotes empty and black full. We might add a black $\mathcal{A}$-tile to move down from situation $\ell$ to situation $\ell+1$ and move the first empty position to the right. We could also view this move as adding a white $\mathcal{A}$-"anti-tile" to move up from $\ell+1$ to $\ell$ and to the left. Thus 3 positions switch from black to white or white to black. Rotate the table $180^{\circ}$ (around the 0 position of line 0 ) and interpret white as full and black as empty. Then the white $\mathcal{A}$-"anti-tile" becomes a white $\mathcal{B}$-tile. The previous move now looks like adding a white $\mathcal{B}$-tile to move down from line $-(\ell+1)$ to line $-\ell$ and one or more positions to the right.

\section{Situations, states, words and duality}

We need, among other things, notational counterparts to the two kinds of diagrams in the tables above. We first exhibit them in an example and then define them. An initially confusing notation turns out to be useful. At any given stage, let $E=\{-e \mid$ position $e$ is not yet covered by a tile $\}$ and call $(F,-E)$ the situation. All the translation invariant properties of the situation $(F,-E)$ are captured by the corresponding state $S=\operatorname{state}(F,-E) \subset[1, \infty)$. This $S$ is the finite set obtained by translating the first empty position to 0 and keeping the finite sequence of empty and full positions. 
Example 2 Consider the situation $\left(F_{5},-E_{5}\right)$ at line 5 in the first table. The sets of full and empty positions are $F_{5}=\{\cdots, 9,10,11,13,14,16\}$. and $-E_{5}=\{12,15,17,18,19, \cdots\}$. The corresponding state is $S_{5}=\{1,2,4\}=\square \mathbf{\square} \square$. Write $\left(F_{4},-E_{4}\right)^{\mathcal{A}}=\left(F_{5},-E_{5}\right)$ since the placement of an $\mathcal{A}$-tile was the move resulting in this situation. Also write $S_{4}^{\mathfrak{A}}=S_{5}$ and $S_{5}^{\mathfrak{A}^{-1}}=S_{4}$ where $S_{4}=\{2\}=\square \square \boldsymbol{D}$. The dual fact is ${\overline{S_{5}}}^{\mathcal{B}}=\overline{S_{4}}$ where $\overline{S_{5}}=\square \square \square=S_{1}$ and $\overline{S_{4}}=\square \mathbf{\square}=S_{2}$. The word $w=\mathcal{A} \mathcal{A B A B B}$ describes the six step cycle of that table; $\emptyset^{w}=\emptyset$. It is self-dual, $w=\bar{w}$. For the six states on that cycle, $\overline{S_{i}}=S_{6-i}$. In particular, $S_{3}$ and $S_{0}=S_{6}$ are self-dual.

Definition 1 A situation is any pair $(F,-E)=(F,-(\mathbb{Z} \backslash F))$ with $F \subset \mathbb{Z}$ and both $f=\max F$ and $e=$ $\min (\mathbb{Z} \backslash F)=\min (-E)=-\max E$ finite. A state is any finite set of positive integers.

- Given a situation $(F,-E)$, the state corresponding to $(F,-E)$ is the finite set $S \subset \mathbb{N}$ with $F-e=$ $(-\infty, 0) \uplus S$

$$
\text { state } F=\operatorname{state}(F,-E)=(F-e) \cap \mathbb{N}=(F \cap(e, f])-e .
$$

- Given a state $S$, the (canonical) situation corresponding to $S$ is the unique situation $(F,-E)$ with state $(F,-E)=S$ and $\min (-E)=0$

$$
\operatorname{sit} S=(\mathbb{Z} \backslash(\mathbb{N} \backslash S),-(\mathbb{N} \backslash S))=((-\infty, 0) \uplus S,-(\mathbb{N} \backslash S))
$$

- Call a state $S$ short if $\max S<b$, moderate if $\max S<n$, and immoderate otherwise. The state $\emptyset$ is short and moderate.

- A situation is short or (im)moderate exactly when its state is; $(F,-E)$ is short when $f-e<b$ and moderate when $f-e<n$.

Lemma 3.1 Let $S=\operatorname{state}(F,-E)$ and $(\widehat{F},-\widehat{E})=\operatorname{sit} S$ then

1. $S=\operatorname{state}(F+x,-(E+x))$ for any $x$.

2. $\max S=\max F-\min (-E)=\max F+\max E$

3. $(F,-E)=(\widehat{F}+e,-(\widehat{E}+e))$ where $e=\min (-E)$

We next define functions $(\cdot)^{C}$ and $(\cdot)^{C^{-1}}$ so that the equation $(J,-K)^{C}=\left(J^{\prime},-K^{\prime}\right)$ describes the result of placing a $C$-tile to fill the first empty space and two others in $(J,-K)$ and $\left(J^{\prime},-K^{\prime}\right)^{C^{-1}}=(J,-K)$ describes the result of removing a $C$-tile (or adding a $C$-"anti-tile") to empty the last full space and two others in $\left(J^{\prime}, K^{\prime}\right)$. For moderate situations, but no others, the two $\mathcal{C}$-tiles are the same and the maps are mutual inverses.

Example 3 Let $\mathcal{A}=\{0,2,5\}=\square \square \square \square$ and consider

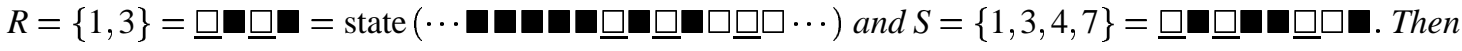

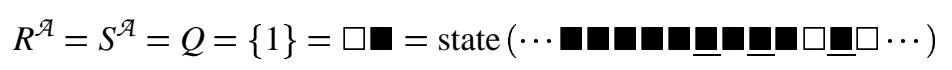

and $Q^{\mathcal{A}^{-1}}=R$.

Check that $S^{\prime}=\{1,3,4,9\}=\square \square \square \square \square \square$ has $Q^{\prime}=S^{\prime \mathcal{A}}=\{3\}=\square \square \square$ but $Q^{\prime \mathcal{A}^{-1}}$ is undefined. None of these problems arise if immoderate states such as $S$ and $S^{\prime}$ are excluded. 
Definition 2 Let $(J,-K)$ and $\left(J^{\prime},-K^{\prime}\right)$ be two situations with $\max J=j, \max J^{\prime}=j^{\prime}, \min -K=k$, and $\min -K^{\prime}=k^{\prime}$ Consider this pair of equations involving a $C$-tile $T$

$$
\begin{aligned}
& \text { 1. }(J,-K)=\left(J,\left(-K^{\prime}\right) \uplus T\right)=\left(J,-\left(K^{\prime} \uplus-T\right)\right) \\
& \text { 2. }\left(J^{\prime},-K^{\prime}\right)=\left(J \uplus T,-K^{\prime}\right)
\end{aligned}
$$

Write

$$
\begin{aligned}
(J,-K)^{\mathcal{C}} & =\left(J^{\prime},-K^{\prime}\right) \text { if they hold for } T=\mathcal{C}+k=\{k, c+k, n+k\} \\
\left(J^{\prime},-K^{\prime}\right)^{\mathcal{C}^{-1}} & =(J,-K) \text { if they hold for } T=\mathcal{C}-n+j^{\prime}=\left\{j^{\prime}-n, j^{\prime}-n+c, j^{\prime}\right\}
\end{aligned}
$$

Also write $S^{C}=S^{\prime}$ or $S^{C^{-1}}=S$ for the corresponding states.

$$
R^{C}=\operatorname{state}\left((\operatorname{sit} R)^{C}\right) \text { and } R^{C^{-1}}=\operatorname{state}\left((\operatorname{sit} R)^{C^{-1}}\right)
$$

Each map has as domain only certain situations and states. For example, $R^{C}$ is defined exactly when $R \cap C=\varnothing$. All this defines a map $(\cdot) \mapsto(\cdot)^{w}$ for each word $w$ in $\langle\mathcal{A}, \mathcal{B}\rangle$, the free non-abelian group generated by the symbols $\mathcal{A}, \mathcal{B}$ (and $\mathcal{A}^{-1}, \mathcal{B}^{-1}$ ). Of course some may have empty domain, see example 4 . To describe a sequence of moves in a tiling, restrict to $(\cdot)^{w}$ for words in the symbols $\mathcal{A}, \mathcal{B}$. That is, words in the free semigroup $\{\mathcal{A}, \mathcal{B}\}^{*}$.

Definition 3 The dual of a situation is obtained by from interchanging left $\leftrightarrow$ right and empty $\leftrightarrow$ full

$$
\overline{(F,-E)}=(E,-F) \text {. }
$$

Then the corresponding states are called duals of each other

$$
\begin{aligned}
& \bar{S}=\operatorname{state}(\overline{\operatorname{sit}(S)}) \text { So, } \\
& \bar{S}=(0, m] \backslash\{m-j \mid j \in S\} \text { where } m=\max S
\end{aligned}
$$

The dual of a word $w$ in the free group $\langle\mathcal{A}, \mathcal{B}\rangle$ is the word $\bar{w}$ obtained by reversing the order and interchanging $\mathcal{A} \longleftrightarrow \mathcal{B}$ and $\mathcal{A}^{-1} \longleftrightarrow \mathcal{B}^{-1}$

$$
\overline{\mathcal{A}}=\mathcal{B}, \quad \overline{\mathcal{B}}=\mathcal{A}, \quad \overline{\mathcal{C}^{-1}}=\bar{C}^{-1}, \quad \overline{u v}=\bar{v} \bar{u} .
$$

The common notation should not cause any confusion. In $\overline{\mathcal{A}}=\mathcal{B}$, the $\mathcal{A}$ is being viewed as a symbol rather than as the set it names. Strictly speaking, that set is not a state, so no other interpretation makes sense. In either setting, $\overline{\overline{(\cdot)}}=(\cdot)$. The next result is clear from the second description of $\bar{S}$, and also from lemma 3.1(2).

Lemma 3.2 For a state $S, \max \bar{S}=\max S$. In particular, both are moderate if either is.

The following theorem, at least for the words $w=\mathcal{A}, \mathcal{B}, \mathcal{A}^{-1}$ and $\mathcal{B}^{-1}$, is fairly obvious from looking at the diagrams above, and even more so from the diagrams below. 
Theorem 1 For states $P$ and $Q$ and any word $w \in\langle\mathcal{A}, \mathcal{B}\rangle$, the following are equivalent in that if one equation has the left hand side defined, all do and all are true. Then $P, Q, \bar{P}, \bar{Q}$ are all moderate.

$$
\begin{aligned}
P \text { is moderate and } P^{w} & =Q \\
Q \text { is moderate and } Q^{w^{-1}} & =P \\
\bar{Q} \text { is moderate and } \bar{Q}^{\bar{w}} & =\bar{P} .
\end{aligned}
$$

Proof. Given the definitions, it is enough to prove this for the four words $w=\mathcal{A}, \mathcal{B}, \mathcal{A}^{-1}, \mathcal{B}^{-1}$. It is equivalent to prove the corresponding equations for situations. We use the notation of definition 2 .

$(J,-K)^{\mathcal{C}}=\left(J^{\prime},-K^{\prime}\right)$ is equivalent to $T=\mathcal{C}+k=\{k, c+k, n+k\}$ satisfying

1. $(J,-K)=\left(J,\left(-K^{\prime}\right) \uplus T\right)=\left(J,-\left(K^{\prime} \uplus-T\right)\right)$ and

2. $\left(J^{\prime},-K^{\prime}\right)=\left(J \uplus T,-K^{\prime}\right)$

Then $(J,-K)$ is moderate exactly if $j<n+k=\max T$, i.e. if $j^{\prime}=\max (J \uplus T)=\max T$. But then $T=C-n+j^{\prime}=\left\{j^{\prime}-n, j^{\prime}-n+c, j^{\prime}\right\}$, so the conditions for $\left(J^{\prime},-K^{\prime}\right)^{C^{-1}}=(J,-K)$ are satisfied. Furthermore, $\left(J^{\prime},-K^{\prime}\right)$ is now moderate if $j^{\prime}=n+k<n+k^{\prime}$. But $k<k^{\prime}$ follows from $-K^{\prime} \subset-K$ with $k \notin-K^{\prime}$.

Conversely, the lone equation $\left(J^{\prime},-K^{\prime}\right)^{C^{-1}}=(J,-K)$ says that 1 . and 2. hold for $T=C-n+j^{\prime}$. Then $\left(J^{\prime}, K^{\prime}\right)$ is moderate exactly if $k^{\prime}>j^{\prime}-n=\min T$, i.e. exactly if $k=\min \left(\left(-K^{\prime}\right) \uplus T\right)=\min T$. But then $T=\{k, c+k, n+k\}$ So $n+k=j^{\prime}$. Here $J \subset J^{\prime}$ but $j^{\prime} \notin J$, so $j<j^{\prime}=n+k$ and this makes $(J,-K)$ moderate.

To finish, first recall that $\bar{C}=-C+n$ and then write an equivalent form of $\left(J^{\prime},-K^{\prime}\right)^{C^{-1}}=(J,-K)$ namely $-T=(-\mathcal{C})+n-\max \left(J^{\prime}\right)=\bar{C}+\min \left(-J^{\prime}\right)$ satisfies

1. $(K,-J)=\left(K^{\prime} \uplus-T,-J\right)$ and

2. $\left(K^{\prime},-J^{\prime}\right)=\left(K^{\prime},-(J \uplus T)\right)=\left(K^{\prime},(-J) \uplus(-T)\right)$.

This is exactly the definition of $\left(K^{\prime},-J^{\prime}\right)^{\bar{C}}=(k,-J)$. The claims about states being moderate now come from the lemma above.

\section{The state digraph $D_{a b}$}

We have said that all the translation invariant properties of the situation $(F,-E)$ are captured by the corresponding state. In particular, all the information about tiling properties of $\{0, a, a+b\}$ and $\{0, b, a+b\}$ is encoded in a finite directed graph.

Definition 4 The digraph $\mathbf{D}_{\mathbf{a b}}$ has the $2^{n-1}$ moderate states $S \subseteq[1, n)$ as nodes with a directed edge labelled $\mathcal{C}$ from $R$ to $R^{C}$ where $\mathcal{C} \in\{\mathcal{A}, \mathcal{B}\}$.

Theorem 1 asserts the useful fact that $R^{C}$ is moderate when $R$ is. Each node $R$ in $D_{a b}$ has 1 or 0 departing edges labelled $\mathcal{A}$. The theorem also asserts that there are 1 or 0 entering edges so labelled, according as $R^{\mathcal{A}^{-1}}$ is or is not defined. Similarly for $\mathcal{B}$. 
Here are diagrams of $D_{23}$ (the example above) and of $D_{14}$. The duality shows up in both cases as the fact that reflection in the central axis interchanges nodes $S \leftrightarrow \bar{S}$ (i.e. left $\leftrightarrow$ right and black $\leftrightarrow$ white) and affects edges by reversing direction and interchanging labels $\mathcal{A} \leftrightarrow \mathcal{B}$.

Consider first the case $(a, b)=(2,3)$. The period $2 \mathbb{Z}$-tiling shows up as the two-cycle at the bottom. The directed hexagon just above it corresponds to the period 6 tiling. In this case, we can tile the interval $[0,3 k)$ exactly for $k=6,8,10, \cdots$. Observe the height function on the right side. An $\mathcal{A}$-edge increases height by 1 while a $\mathcal{B}$-edge lowers it by 1 . In particular, the graph is bipartite. There are two connected components (if we ignore edge direction) and only one has a cycle. There are 7 nodes which can be part of an bi-infinite path.

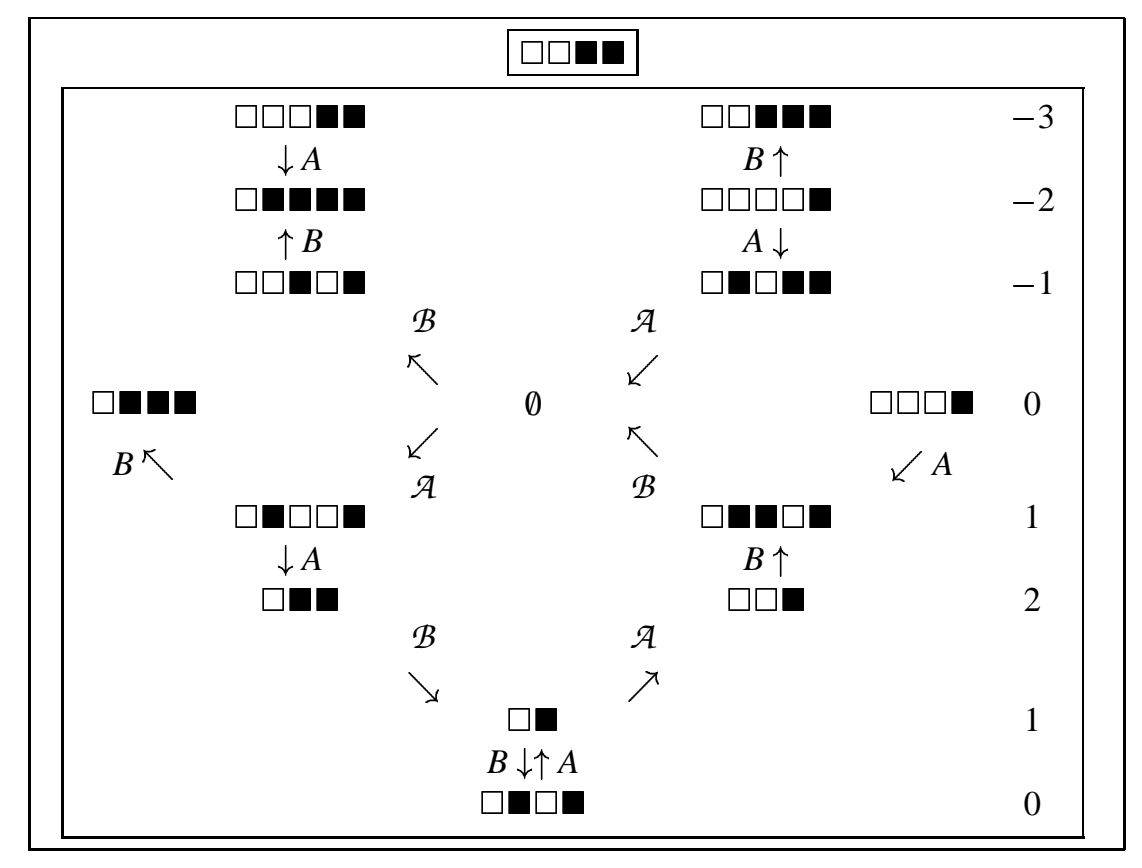

$\left(D_{23}\right)$

In the next graph, $D_{14}$, there are loops and 3-cycles. Hence, no height function as above is possible. There are 6 connected components and 4 contain cycles (including loops). The labels are an invariant 
described in definition 6 .

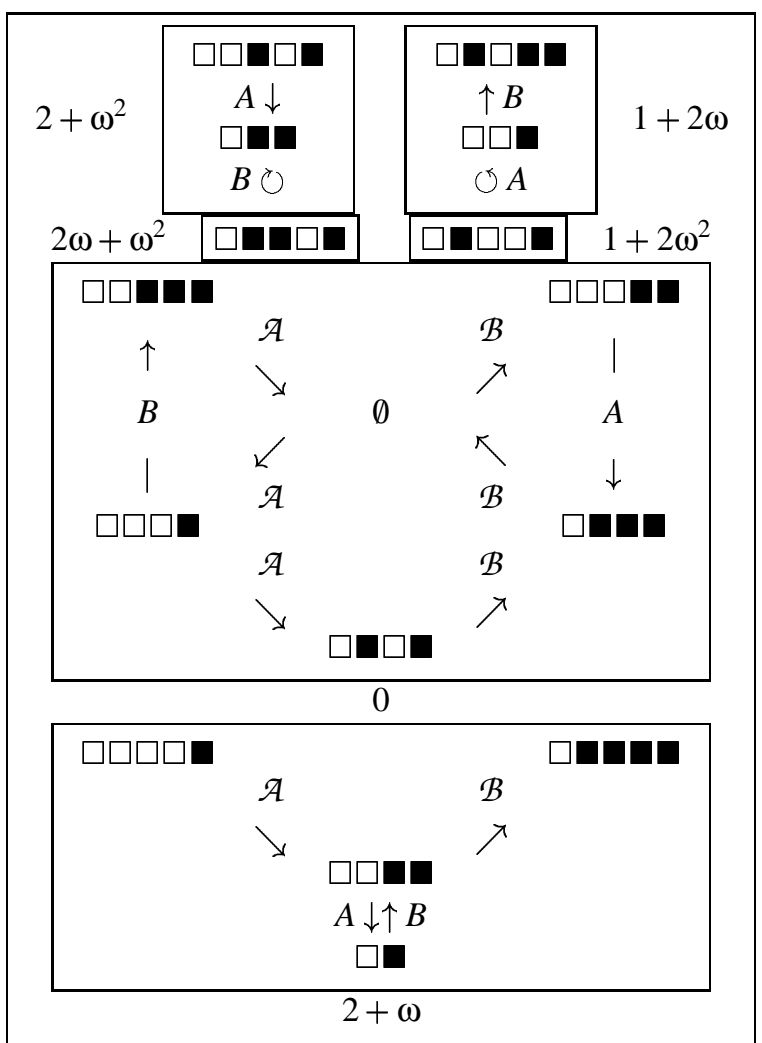

$\left(D_{14}\right)$

We can study tilings and the states which can occur in them utilizing the corresponding paths in $D_{a b}$. An interval tiling corresponds to a closed path from $\emptyset$ to itself, a $\mathbb{Z}_{m}$-tiling to a closed path, an $\mathbb{N}$-tiling to an infinite path starting at $\emptyset$,and a $\mathbb{Z}$-tiling to a bi-infinite path.

Definition 5 Call $S$ an $\mathbb{I}$-state, $R$-state, $\mathbb{N}$-state, or $\mathbb{Z}$-state respectively when it is a state in a tiling/path of the appropriate type.

An R-state is Recurrent. Note that the state $\square \square \square$ is totally isolated in $D_{23}$ but is a $\mathbb{Z}$-state in $D_{14}$. The properties of a given state are only meaningful in relation to the particular values of $a$ and $b$.

Before going on we give a promised example

Example 4 In $D_{23}$,. $S \mapsto S^{\mathcal{A} \mathcal{A}}$ has 3 states in its domain and $S \mapsto S^{\mathscr{A} \mathcal{A} \mathcal{A} \mathfrak{A}}$ has one and $S \mapsto S^{\mathscr{A} \mathcal{A} \mathcal{A} \mathcal{A} \mathcal{A}}$ has none.

Remark 4.1 For the tiling properties above, $\mathbb{I} \Rightarrow \mathbb{N}$. Also, $R \Rightarrow \mathbb{Z}$ since a closed path can be repeated in both directions to give a two way infinite path. Furthermore, $\mathbb{N} \Rightarrow \mathbb{Z}$ since we may take an $\mathbb{N}$-tiling containing the given state, reflect it to get a tiling of the negative integers, and combine the two to get a $\mathbb{Z}$-tiling. Formally, if the $\mathbb{N}$-tiling is $\mathcal{A} \oplus P_{\mathcal{A}} \biguplus \mathcal{B} \oplus P_{\mathcal{B}}=\mathbb{N}$ then

$$
\mathcal{A} \oplus\left(P_{\mathfrak{A}} \uplus\left(-P_{\mathcal{B}}-n-1\right)\right) \biguplus \mathcal{B} \oplus\left(P_{\mathcal{B}} \uplus\left(-P_{\mathfrak{A}}-n-1\right)\right)=\mathbb{Z} .
$$


Define a relation on $\mathbb{Z}$-states $S_{1} \succsim S_{2}$ if there is a path from $S_{1}$ to $S_{2}$ and $S_{1} \approx S_{2}$ if there is a cycle containing both.

Conjecture 1 For any given $a$ and $b$,

- $S_{1} \succsim S_{2}$ implies $S_{1} \approx S_{2}$. This would imply that every $\mathbb{N}$-state is an $\mathbb{I}$-state.

- Every $\mathbb{Z}$-state is an $R$-state.

Conjecture 2 Given any single integer prototile and its reflection, if there is an $\mathbb{N}$ tiling then there is an interval tiling. Furthermore, every $\mathbb{N}$-state is an $\mathbb{I}$-state and every $\mathbb{Z}$-state is an $R$-state.

\section{Further structure}

Examination of $D_{a b}$ for various small $a$ and $b$ lead to many of the theorems and conjectures of this paper. A component of $D_{a b}$ is a connected component of the corresponding undirected graph (perhaps just for the induced graph on the R-states). In this section we examine the number of components. First, though, we give a small problem.

Assign each edge $S \rightarrow S^{C}$ in $D_{a b}$ the length $|S|-\left|S^{C}\right|+3$, that is, the first number not in $S \uplus C$. Then the total length of a closed cycle is $3 t$ where $t$ is the number of edges. In a tiling the positions fall into equivalence classes mod 3 . To standardize at least the $\mathbb{N}$-situations, say that the first empty position in an $\emptyset$-situation is type 0 . Then state $(F,-E)$ determines $\max F \bmod 3$.

Problem 3 Give a procedure to find the congruence class corresponding to a position in a given state. Equivalently, a procedure to find the total length $\bmod 3$ of any path from one given state to another.

Consider three cases

case $\mathbf{I} \operatorname{gcd}(a, b)=1$ and $a \neq b \bmod 3$

case II $\operatorname{gcd}(a, b)=1$ and $a=b \bmod 3$

case III $\operatorname{gcd}(a, b)=d>1$

Conjecture 4 In case I all R-states occur in the same component. Furthermore

1. Any $\mathbb{Z}$-state may be reached from any other. In particular, every $\mathbb{Z}$-state is an $\mathbb{I}$-state.

2. There is a height function $h$ on all the states such that $h\left(S^{\mathcal{A}}\right)=h(S)+1$ and $h\left(S^{\mathcal{B}}\right)=h(S)-1$. In particular, $D_{a b}$ is bipartite and every periodic tiling has period a multiple of 6 . Moreover, each cycle has equal numbers of $\mathcal{A}$-edges and $\mathcal{B}$-edges.

3. There is a $K=K_{a b}$ such that for every $k \geq K$ there is a tiling of $[0,6 k)$.

Problem 5 Short of a proof, it would be interesting to conjecture a formula (depending on a and $b$ ) for computing (relative) height. Simply to conjecture a formula for determining parity would be nice. The problem above of finding path lengths mod 3 would thus be refined to finding length mod 6 . The case $a=1$ might be good to study. See claim 7.1. 
For a height function we would need to decree a height for some state in each component. It would be reasonable to set the lowest elements of each component at height 0 . However, it is tempting to set $h(\emptyset)=0$ in its component. Of course conjecture 8 is that this is the only component with $\mathbb{Z}$-states.

We will show that there are many connected components in the other two cases. The method is to define an invariant which is constant on components and to show it has several values. Often the partition into equivalence classes according to the value of the invariant is much coarser than that according to components. Thus we do not try for the best possible bound on the number of values the invariant takes on. The main point is that, unlike the conjectured result for case I, there are definitely many components in the other cases.

Given a finite set $C \subset \mathbb{Z}$, let $C(x)$ denote the $\operatorname{sum} \sum_{i \in C} x^{i}$. If $\operatorname{gcd}(a, b)=1$ and $a=b \bmod 3$ then any tile $T=\{e, e+a, e+a+b\}$ or $T=\{e, e+b, e+a+b\}$ is a set of residues mod 3. Hence $T(\omega)=0$ for any tile $T$ where $\omega$ is a primitive cube root of unity. This suggests using $C(\omega)$. We can't simply use $S(\omega)$ as an invariant on states because of the way we truncate full spaces left of the first empty space. As it stands, $S=\emptyset$ has $S(\omega)=0$ but $S=\emptyset^{\mathcal{B}}=\{b-1, a+b-1\}$ does not. We start with situations.

Definition 6 Given a situation $(F,-E)$ define $\sigma(F,-E)=\sigma F=\widetilde{F}(\omega)$ where $\widetilde{F}=F-c \cap[0, \infty)$ and $c \leq \min -E$ is chosen so that $\widetilde{F}$ has cardinality a multiple of 3 . For a state $S$ define $\sigma S=\sigma \operatorname{sit} S$.

The actual choice of $c$ does not matter. If $c-3$ is used in place of $c$, the result is to change each previous summand $\omega^{i}$ to $\omega^{3} \omega^{i}=1 \omega^{i}$ and include three more summands $1+\omega+\omega^{2}=0$. Note that $\sigma F=\sigma(F+x)$. The diagram for $D_{14}$ is decorated with this invariant.

Theorem 2 For case II, let $\sigma$ be as above, then

- for every state $R, \sigma R=\sigma R^{C}$ provided $R^{C}$ is defined.

- The number of $\approx$-equivalence classes is at least $\left(\frac{b}{3}\right)^{2}$.

Proof. It is easier to use situations. $(F,-E)^{C}=(F \uplus T,-(E \backslash T))$. The same value of $c$ used for $\sigma F$ can be used again so $\sigma(F \uplus T)=\sigma F+T(\omega)=\sigma F+0$. We will later see that every short state is an $\mathrm{R}$-state, so a lower bound is the number of possible values $\sigma(S)$ for $\max (S)<b$. Since $\omega^{2}=-1-\omega$, it suffices to look at cases where all members of $\widetilde{F}$ miss one of the three congruence classes mod 3 . Then the value only depends on how many members of each of the other two classes are chosen. There are three ways to choose the excluded class and then about $\frac{1}{3}\left(\frac{b}{3}\right)^{2}$ choices in each case. The $\frac{1}{3}$ accounts for the fact that the two numbers chosen must add to a multiple of 3 .

It should be easy to show that the invariant takes on $q^{2}$ values if $b=3 q-3$ or $3 q-2$ and $q^{2}+q$ if $b=3 q-1$. From limited computations this appears to be a very weak lower bound for all but the smallest cases. In particular, it appears possible that there are distinct components with the same invariant whenever $a+b>8$.

To just see that there is more than one component we could identify a couple of very small components.

Theorem 3 In case II there are two loops corresponding to the tilings $\mathcal{C} \oplus 3 \mathbb{Z}=\mathbb{Z}$. Each is on the only $R$-state of its component. In particular, $D_{a b}$ is not bipartite.

Proof. Consider the tiling $\mathcal{A} \oplus 3 \mathbb{Z}=\mathbb{Z}$. Assume that $a=3 \alpha+1 \geq 1$ and $b=3 \beta+1 \geq 4$ (the other case is essentially the same.) The state is always that of $\mathcal{A} \oplus\{\cdots,-9,-6,-3\}$. Then the next tile will be $\{0, a, a+b\}=\{0,3 \alpha+1,3(\alpha+\beta)+2\}$ and the state is 
$S=\{1,4,7, \cdots, 3 \alpha-2\} \uplus\{2,5,8, \cdots, 3(\alpha+\beta)-1\}$. We can make the move $S^{\mathcal{B}}$ of adding instead $\{0,3 \beta+1,3(\alpha+\beta)-2\}$ but this leads us to a dead-end since eventually the first empty space is at $3 \alpha+1$ and the choices for the next tile will be

$\{3 \alpha+1,3(\alpha+\gamma)+2,3(2 \alpha+\beta)+2\}$ with $\gamma=a$ or $\beta$. But that middle position was already filled by the end of a tile $\mathcal{A}+3(\gamma-\beta)$. Note that $S^{\mathcal{B}^{-1}}$ is undefined.

By duality, the state for the tiling $\mathcal{B} \oplus 3 \mathbb{Z}=\mathbb{Z}$ has $S^{\mathcal{A}}$ undefined. There could be a directed path leading into it but it would be of bounded length.

Theorem 4 In case III, the number of components at least $\frac{3^{d-1}}{d}$.

Proof sketch. If $\operatorname{gcd}(a, b)=d$ then every tile lies completely in one congruence class mod $d$. Hence a sequence of situations in $D_{a b}$ corresponds to having $d$ copies of $D_{a^{\prime} b^{\prime}}$ where $\left(a^{\prime}, b^{\prime}\right)=\left(\frac{a}{d}, \frac{b}{d}\right)$ and at step $d q+r$ moving from the current situation $\left(F^{r},-E^{r}\right)$ in copy $r$ to either $\left(F^{r},-E^{r}\right)^{\mathcal{A}}$ or $\left(F^{r},-E^{r}\right)^{\mathcal{B}}$. Restrict attention to those cases where all $r$ situations are $\mathbb{I}$-situations. Given such a compound situation, relax the rule about going in order and separately extend each situation to a situation with corresponding state $\emptyset$; One with $\max F^{r}=\min \left(-E^{r}\right)-1$. Then record the $d$ numbers $\max \left(F^{r}\right)-\max \left(F^{r+1}\right) \bmod 3$. The list does not depend on the actual value $\max \left(F^{r}\right)$ since the potential values for that number all differ by multiples of 3. We don't know which number comes from which congruence class; only which belong to consecutive classes. Hence we have a circular list of length $d$ with entries from $\{0,1,2\}$ and sum $0 \bmod 3$. There are $3^{d-1}$ linear lists of this type and each circular list corresponds to $d$, or maybe fewer, linear lists.

Looked at another way, we noted at the start of this section that the mod 3 class of a position is meaningful in the context of states. We essentially have $d$ non-interacting interlaced copies of $\mathbb{Z}$. Each has a standard labelling $\cdots 012012012 \cdots$ of its positions determined by the current state. We are examining the relative phases of consecutive copies.

Polya counting of bracelets could improve the bound. Again, the bound seems likely to be quite weak. If $a^{\prime} \neq b^{\prime} \bmod 3$ then, if conjecture 8 is true, the number would be more on the order of $\frac{6^{d-1}}{d}$. If $a^{\prime}=b^{\prime} \bmod 3$ then aside from the issue of relative phase, there are many equivalence classes of states. The number would be more on the order of $\frac{(3 k)^{d-1}}{d}$, where $k>\left(\frac{b}{3}\right)^{2}$ is the number of components in $D_{a^{\prime} b^{\prime}}$.

Problem 6 Find an easier way to compute this invariant.

\section{The greedy algorithm}

Some of the nodes (states) of $D_{a b}$ have no edges leaving them. In fact this is true of a quarter of them; Those with $\{a, b\} \subset S$ have this dead-end status. Others may have one or two outwardly directed edges, yet have them lead to only to dead-end states.

Consider the following greedy rule for generating a path of length 0 or more starting at a given state in $D_{a b}$

Use $\mathcal{A}$ if you can. Otherwise, use $\mathcal{B}$.

In other words, when at a dead-end, a state with no outward edges, give up. When there is only one departing edge, use it. When there is both an outward $\mathcal{A}$-edge and an outward $\mathcal{B}$-edge, use the $\mathcal{A}$-edge. It turns out that the greedy rule started at state $S=\emptyset$, produces a tiling of an interval $[0,3 x)$ with $x=x_{a b} \leq$ $2^{a+b-1}$. 
There are various strategies for proving this. One is to do a detailed analysis of exactly what the tiling is $[\mathrm{Me}]$. The behavior has an number of interesting features. Another is an easy proof by contradiction. Assuming that we get to a dead-end implies that we failed to follow the rule at an earlier stage. This proof does not provide much information about the length of the interval tiled by the greedy rule. The structure of the tilings produced is of independent interest. There are usually far shorter interval tilings. However, this rule turns out to be very useful for studying $D_{a b}$.

\subsection{Translation without refutation}

This is one of the cases where converting to a direct proof guides one to a stronger result. We briefly show this process.

Given a $\mathbb{Z}$-tiling, like those of example 1 , and a distinguished tile $T_{0}$, there is a unique ordering $\ldots, T_{-2}, T_{-1}, T_{0}, T_{1}, T_{2}, \ldots$ of the tiles such that $T_{i}=\mathcal{C}+e_{i}=\left\{e_{i}, e_{i}+c, e_{i}+n\right\}$ where $e_{i}<e_{j}$ for $i<j$ and $c=a$ or $b$ according as $\mathcal{C}$ is $\mathcal{A}$ or $\mathcal{B}$.

Notice that in $D_{23}$ a path can start with a move from the node $\square \square \square \square \mathbf{\square}=\{4\}$ to $\square \square \square \square=\{1,3,4\}$ and then continue around the graph. However, there are no edges into $\square \square \square \square \square$. To study all of $D_{a b}$, including nodes like this, define a tiling (or partial tiling) of $\mathbb{Z}$ with initial situation $\left(F_{0},-E_{0}\right)$ to be a tiling corresponding to an infinite (or finite) path in $D_{a b}$ starting at the node $S_{0}=\operatorname{state}\left(F_{0},-E_{0}\right)$. Thus an $\mathbb{N}$-tiling is a $\mathbb{Z}$-tiling with initial situation $\left(F_{0},-E_{0}\right)=\operatorname{sit} \emptyset=(\mathbb{Z} \backslash \mathbb{N},-\mathbb{N})$.

Definition 7 Let $F_{g}=F_{0} \uplus \biguplus_{\ell<g} T_{\ell}$ be the set of $\boldsymbol{F}$ illed positions and $-E_{g}=\mathbb{Z} \backslash F_{g}$ be the set of Empty positions before the use of $T_{g}$ so $\left(F_{g+1},-E_{g+1}\right)$ is the resulting situation. Say that position $j$ is full at stage $g$ if $j \in F_{g}$ and that position $j$ is empty at stage $g$ if $j \in E_{g}$. A full position not in $F_{0}$ is a start, end or middle according as it is $e_{\ell}, a+b+e_{\ell}$ or the middle element of the appropriate tile $T_{\ell}$. Thus

$$
\begin{aligned}
k \text { is } a \text { start } & \Longleftrightarrow k+a+b \text { is an end } \\
j \text { is an end } & \Longleftrightarrow j-a-b \text { is a start. }
\end{aligned}
$$

Remark 6.1 Suppose that we have a partial tiling $T_{0}, T_{1}, T_{2}, \ldots, T_{g-1}$ with initial state $S_{0}$. Then, as we get ready to try to choose $T_{g}$, any position $j \notin F_{0}$ is either empty or part of tile $T_{i}$. In the latter case, $i<g$ so $\min T_{i}=e_{i}<e_{g}$. Thus,

$$
\begin{gathered}
\text { position } e_{g} \text { is empty } \\
j \geq e_{g} \Rightarrow \text { position } j \text { is not a start } \\
j \geq e_{g}+b \Rightarrow \text { position } j \text { is neither a start nor a middle } \\
j \geq e_{g}+a+b \Rightarrow \text { position } j \text { is empty }
\end{gathered}
$$

Say that the choice $T_{g}=\mathcal{C}+e_{g}$ is blocked if $\mathcal{C}+e_{g} \varsubsetneqq E_{g}$ 
Initialize $\mathcal{A}=\{0, a, a+b\}, \mathcal{B}=\{0, b, a+b\}$

$$
i=0, E_{0}=-\left(\mathbb{N} \backslash S_{0}\right), e_{0}=\min \left(-E_{0}\right), F_{0}=\mathbb{Z} \backslash\left(-E_{0}\right)
$$

(Greedy Algorithm)

Repeat

Choose $T_{i}=\left\{\begin{array}{l}e_{i}+\mathcal{A} \text { if }\left(e_{i}+\mathcal{A}\right) \subset-E_{i} \\ e_{i}+\mathcal{B} \text { else }\end{array}\right.$

HALT with an error if $T_{i} \cap F_{i} \neq \emptyset$

Set $F_{i+1}=F_{i} \uplus T_{i}, E_{i+1}=-\left(\mathbb{Z} \backslash F_{i+1}\right), e_{i+1}=\min \left(-E_{i+1}\right)$

Set $i=i+1$

Forever

Claim 6.1 Repeated application of the greedy rule greedy algorithm, initialized with $S_{0}=\emptyset$, will tile $\mathbb{N}$.

Proof. Unless the algorithm halts, it produces an $\mathbb{N}$-tiling. We will suppose that it halts after completing $g \geq 0$ passages through the loop and obtain a contradiction. It halts exactly when both the first choice, $T_{g}=\overline{\mathcal{A}}+e_{g}$, and the other, $T_{g}=\mathcal{B}+e_{g}$ are blocked. From (7) and (10), $e_{g}$ and $a+b+e_{g}$ are empty. So we are assuming that both $a+e_{i}$ and $b+e_{i}$ are already full. From (9) $j=b+e_{i}$ is neither a start nor a middle So it must be the end of some already specified tile $T_{\ell}$ that ends at $j=b+e$ and starts at $e_{\ell}=j-a-b=$ $e-a$. The rule required us to choose

$$
T_{\ell}=\{0, a, a+b\}+e_{\ell}=\{e-a, e, e+b\}
$$

if possible. But $e$ is an empty position. This is the contradiction.

Before proceeding to a direct proof we observe that a couple of other improvements can be made and show a question answered by the direct proof. First, no use is made of the assumption that $T_{i}=e_{i}+\mathcal{B}$ is blocked. Hence the choice $T_{i}=e_{i}+\mathcal{B}$ is never blocked, provided we refrain from actually making that choice unless forced. Also, the assumption that the initial state is $F_{0}=\emptyset$ is only used when we assert that remark 26 applies to $j=e_{i}+b$ in as much as it satisfies the requirement $j \notin F_{0}$. It suffices that $\max S_{0}<b$, i.e. that $S_{0}$ be short, Change the proof to read

$j=b+e_{i}$ is neither a start, nor a middle, nor an element of $F_{0}$ to obtain.

Corollary 6.2 Initialize the greedy algorithm with any short state $S_{0}$. Then, at the start of each passage through the loop, the following loop invariants are satisfied

1. $e_{i}+b$ is empty.

2. $f_{i}-n+a=f_{i}-b$ is full.

It follows that the algorithm will tile $\mathbb{Z}$ starting with $S_{0}$.

Proof. We have just observed that 1 is a loop invariant. That alone is enough prove the correctness claim. 
For $i=0$, the condition $\max S_{0}<b$ is enough for 2. and position $f_{0}-b<0$ is full as part of $F_{0}$. Otherwise, the previous tile, $T_{i-1}$, filled positions $e_{i-1}, e_{i-1}+n=f_{i}$ and also $e_{i-1}+a=f_{i}-b$, unless that position was already full before that stage. In any case, $f_{i}-b$ is full.

This corollary, with an additional observation, allows us to prove, among other things, the stronger fact that every three element set tiles some finite interval. In fact, the greedy algorithm tiles $\mathbb{N}$ periodically by repeatedly tiling finite intervals.

Lemma 6.3 Initialize the greedy algorithm with a short state and consider the resulting sequence of states. The dual greedy rule

$$
\text { The state } R \text { preceding } S \text { is } S^{\mathcal{B}^{-1}} \text { if defined, otherwise } S^{\mathcal{A}^{-1}} \text {. }
$$

reverses the greedy rule

$$
\text { The state } S \text { following } R \text { is } R^{\mathcal{A}} \text { if defined, otherwise } R^{\mathcal{B}} \text {. }
$$

Proof. Let $S=R^{C}$. We know that $b \notin R$. Thus $\mathcal{C}=\mathcal{B} \Longleftrightarrow n-a=b \in R \uplus \mathcal{C} \Longleftrightarrow \max S-n+b \in$ $S \Longleftrightarrow S^{\mathcal{B}^{-1}}$ is defined.

Theorem 5 Start with any short state $S_{0}=\left(F_{0},-E_{0}\right)$, so $\max F_{0}-\min \left(-E_{0}\right)<b$, and use the greedy rule. After some number $x \leq 2^{a+b-1}$ of steps, the state corresponding to $F_{x}$ will again be $S_{0}$. In particular,

1. The greedy rule for $\{0, a, a+b\}$ and $\{0, b, a+b\}$, applied starting with state $S_{0}=\emptyset$, produces $a$ tiling of an interval $[0,3 x)$ with $x=x_{a b} \leq 2^{a+b-1}$.

2. The greedy rule for $\{0, a, a+b\}$ and $\{0, b, a+b\}$, applied starting with a short state $S_{0}$, produces a tiling of $\mathbb{Z}_{3 x}$ for $x=x_{a, b, F_{0}} \leq 2^{a+b-1}$ and hence a periodic tiling of $\mathbb{Z}$ which returns to the state $S$ every $x$ steps.

Proof. Consider the sequence of states (nodes in $D_{a b}$ ) $S_{0}, S_{1}, \ldots$ arising from the algorithm. Form the graph $D^{\prime}$ with only these nodes and the edges $\left(S_{i}, S_{i+1}\right)$ assigned by the greedy rule. Every node has outdegree 1 in $D^{\prime}$. We have just remarked that each also has indegree one. As the graph is finite, $D^{\prime}$ is a cycle and the result follows.

Question Which of the $2^{a+b-1}$ moderate states can occur in a tiling of the line? (Answer: see Theorem 7)

We have just seen that any of the $2^{b-1}$ short states can. Suppose $a>1$. Half of the $2^{b-1}$ short states, $S$, have position 1 empty. The greedy algorithm sends $S$ to $R=S^{\mathcal{A}}$ with $\max R=a+b-1$. The state $R$ still satisfies the loop invariant " $e_{i}+b$ is empty" but this is justified by corollary 6.2 , not by $R$ being short. The answer emerges from the effort to get a direct proof.

Clearly there is a more general sufficient property than short for belonging to a greedy cycle. The proofs by contradiction rely on the fact that the greedy rule applied to moderate states preserves this property

If $e$ is the first empty position, then $e+b$ is empty as well. 
This property is enjoyed by every short initial state and implies that we are never blocked from following $S$ with $S^{\mathcal{B}}$, provided that we refrain from actually making that move unless forced.

The invariant which allows a direct proof is

$$
\text { If } p \text { is any empty position, then both } p+b \text { and } p+a+b \text { are empty as well. }
$$

With both conditions we can let $S$ be immoderate, although well known results force $\max S \leq(a-1)(a+b-1)-1$ for $\operatorname{gcd}(a, b)=1$. The loop invariant can be altered to restrict to moderate states.

Let tail $S=S \cap[0, a)$ and head $S=S \cap[b, \infty)$. So $S$ is a short state are exactly if head $S=\emptyset$.

Definition 8 Call a state $S$ secure if head $S-b \subseteq \operatorname{tail} S$

This forces $S$ to be moderate since head $S-b \subseteq$ tail $S \subseteq[0, a)$ and also forces $b \notin S$ since $0 \notin$ tail $S$. An equivalent definition is that $S$ is moderate and $q-b$ is never empty when $q$ is full.

Lemma 6.4 Of the $2^{a+b-1}$ moderate states, exactly $3^{a-1} 2^{b-a}$ are secure.

Proof. The positions $j \in[a, b)$ can be chosen in any of the $2^{b-a}$ ways. The pairs of positions $(q, q+b)$ for $q \in(0, a)$ are of three potential types: both empty, $q$ full but with $q+b$ empty, and both full.

Theorem 6 Start with any secure state $S_{0}$ and use the greedy rule. After some number $x \leq 3^{a-1} 2^{b-a}$ of steps, the state corresponding to $F_{x}$ will again be $S_{0}$.

Proof. The greedy rule is to find the least empty position $e$, fill positions $e, e+n$ and either $e+a$ if it is empty or $e+b$ if $e+a$ is already full. Because $S$ is secure we are never blocked from the second choice. Either way, $e+a$ is full when we are done. Now we did not change the truth of "if $x$ is full, so is $x-b$ " for any previously full position. The only position we may have contributed to the head is $j=e+n$. Since $j-b=e+a$ is full, this new state is secure.

We will call this cycle the greedy cycle containing $S_{0}$. A priori, there might be one very long cycle including most of the secure states.

Theorem 7 The states on greedy cycles are precisely the secure states.

Proof. From Theorem 6, every secure state is on a greedy cycle. Let $S$ be a state on a greedy cycle and let $\operatorname{sit} S=(F,-E)$. so $0=\min (-E)$ and every position in $F$ is part of a tile $T=C+x$ with $x<0$. Let $j \in \operatorname{head}(S)$ then $j \geq b$. We need only show that $j-b \in S$. Since $j$ is too big to be $x+a$ or $x+b$ with $x<0$, it must be the end of a tile $T=\mathcal{C}+x=\mathcal{C}+j-n=\{j-n, j-n+c, j\}$. Either this tile is $\mathcal{A}+x=\{j-n, j-n+a, j\}$ or position $x+a=j-n+a$ was already full before we chose $T$. Either way, position $j-n+a=j-b$ is full.

\section{Analysis of the greedy tiling}

The following result appears in [Me]

Theorem 8 Suppose $\operatorname{gcd}(a, b)=1$ with $b>3 a$ and write $b=2 q a+r$ or $b=2 q a-r$ where $0<r<a$. Then $x_{a b}=a(b+r)$

- If $b=2 q a+r$ then $x_{a b}=a(b+r)=2\left(q a^{2}+a r\right)$ 
- If $b=2 q a-r$ then $x_{a b}=a(b+r)=2 q a^{2}$

Example 5 The table below shows the first few values of $x_{5 b}$. If the columns are numbered 1,2,3, 4, 5, 6 $, 7,8,9$ then for $j \neq 5$, the $(i, j)$ entry is $x_{5 b}$ for $b=10 i+j-5$. So $x_{58}=40$ and $x_{524}=140$.

$\begin{array}{ccccccccc}30 & 64 & 40 & 64 & * & 280 & 310 & 230 & 370 \\ 100 & 100 & 100 & 100 & * & 110 & 120 & 130 & 140 \\ 150 & 150 & 150 & 150 & * & 160 & 170 & 180 & 190 \\ 200 & 200 & 200 & 200 & * & 210 & 220 & 230 & 240 \\ 250 & 250 & 250 & 250 & * & 260 & 270 & 280 & 290\end{array}$

Example 6 The values of $x_{25} b$ for $b=118,168,218,268,318 \ldots$ are $3400,4650,5900,7150,8400 \ldots$ The jump is always $2 a^{2}=1250$ and the tilings follow a common pattern. But $x_{2568} \neq 2150$, the value that fits the pattern. As it turns out, $x_{2568}=1298600$. If the common pattern is used for $b=68$, a valid tiling using 2150 tiles does result. However, in this case it is not the greedy tiling.

Problem 7 Analyze the number $x_{a b}$, and more generally, the greedy interval tiling, for $a<b<3 a$.

The case $a=1$ is instructive.

Claim 7.1 Consider the $2^{b}$ states $R \in D_{1 b}$

- One quarter of them are "dead-end" states with no outgoing directed edges, those with $\{1, b\} \subset R$.

- Dually, one quarter of them are "garden of Eden" states with no incoming edges, those with $b \in S$ but $b-1 \notin R$

- On eighth of them are both garden of Eden and dead-end states, those with $\{1, b\} \subset R$ but $b-1 \notin R$.

- The $\mathbb{Z}$-states are exactly the remaining $5 \cdot 2^{b-3}$ states.

Proof. The first three parts are routine. Half of the states have $\max R<b$, they are short and thus belong to a greedy cycle. It remains only to consider the $2^{b-3}$ states with $\{b-1, b\} \subset R$. but $1 \notin R$. For such an $R, Q=R^{\mathcal{B}^{-1}}$ is defined and is short as is $S=R^{\mathcal{A}}$. Then there is a $\mathbb{Z}$ tiling including $R$ which goes from $R$ to $S$ and then around its greedy cycle and, traced backwards from $R$ goes back to $Q$ and around its greedy cycle.

Problem 8 At least for $a=1$ show that all the $\mathbb{Z}$-states are $R$-states. Also, show that every secure state can be reached from $\emptyset$ provided $a+b \bmod 3 \neq 2$.

\section{Duality and the greedy rule.}

The nodes of $D_{a b}$ along with only those edges selected by the greedy rule is a discrete dynamic system. The recurrent states are the secure states (we might or might not restrict to only these states.) From the pigeon hole principle we can deduce that the greedy algorithm, started at any secure state, forms a cycle of length no more than $3^{a-1} 2^{b-a}$.

Problem 9 Investigate the distribution of cycle sizes. Describe the greedy cycle corresponding to some class of states, for example the self-dual secure states. 
We will show here, among other things, that a greedy cycle contains 0 or 2 self dual states. This alone forces there to be many greedy cycles.

Recall that $D_{a b}$ has the $2^{a+b-1}=2^{n-1}$ moderate states as nodes. There are $3^{a-1} 2^{b-a}$ secure states, including the $2^{b}$ short states. If $b=2 j$ or $2 j+1$ then there are exactly $2^{j}$ self-dual short states. This gives a lower bound of $2^{j-1}$ for the number of greedy cycles. The task of enumerating self-dual secure states is somewhat less routine. It would give a better lower bound, but it is not clear how tight this would be.

Lemma 8.1 If $Q$ is a secure state, so is $\bar{Q}$

Proof. The condition is that there be no pairs $(i, i+b)$ with $i \notin Q$ but $i+b \in Q$. Let $m=\max Q$. This forbidden circumstance is equivalent to $m-i-b \notin \bar{Q}$ but $m-i \in \bar{Q}$.

Lemma 8.2 Let $Q$ be a secure state

1. Suppose that the greedy rule sends $Q$ to $R=Q^{C}$, then it sends $\bar{R}$ to $\bar{Q}=\bar{R}^{\bar{C}}$.

2. Let $w=\mathcal{C}_{1} \mathcal{C}_{2} \cdots \mathcal{C}_{k}$ be the word in $\{\mathcal{A}, \mathcal{B}\}^{*}$ which describes the greedy cycle $Q=Q_{0}, Q_{1}, \cdots$, $Q_{k-1}, Q_{k}=Q$. So each $\mathcal{C}_{i}$ is $\mathcal{A}$ or $\mathcal{B}$ and $Q_{i-1}^{C_{i}}=Q_{i}, Q^{w}=Q$. Then $\bar{Q}^{\bar{w}}=\bar{Q}$ and $\bar{w}$ describes the greedy cycle $\bar{w}=\bar{C}_{k} \bar{C}_{k-1} \cdots \bar{C}_{1}$ with $\overline{Q_{i}} \bar{C}_{i}=\overline{Q_{i-1}}$ and $\bar{Q}^{\bar{w}}=\bar{Q}$.

Proof. Since $0 \notin Q$ and $Q$ is secure, $b \notin Q$.

If $R=Q^{\mathcal{A}}$ then $n=\max Q \uplus \mathcal{A}$ and $a \in Q \uplus \mathcal{A}$ but $b \notin Q \uplus \mathcal{A}$. Let $m=\max Q^{\mathcal{A}}=\max R$. Then $a+m-n \in R$ but $b+m-n \notin R$. Thus $m-(a+m-n)=n-a=b \notin \bar{R}$ but $m-(b+m-n)=n-b=a \in \bar{R}$. This means that the greedy rule sends $\bar{R}$ to $\bar{R}^{\mathcal{B}}$ as desired.

It remains, for 1., to consider the case $R=Q^{\mathcal{B}}$. Then $n=\max Q \uplus \mathcal{B}$ and $b \in Q \uplus \mathcal{B}$. Again let $m=\max R$. Then $m-a=b+m-n \in R$ so $a=m-(m-a) \notin R$. This means that the greedy rule sends $\bar{R}$ to $\bar{R}^{\mathcal{A}}$ as desired.

It follows by induction on $k$ that the greedy cycle of $\bar{Q}$ is as stated.

Lemma 8.3 Let $S$ be a self-dual secure state and consider the greedy cycle of $S_{0}=S$.

- The word $w$ which describes this cycle $S^{w}=S$ is self-dual; $w=u \bar{u}=\bar{w}$.

- The word $w$ has even length, $k=2 j$ and as many $\mathcal{A}$ 's as $\mathcal{B}$ 's.

- $\overline{S_{i}}=S_{2 j-i}$ for $0 \leq i \leq 2 j$

- The opposite node of the cycle, $S^{u}=S_{j}$, is also self-dual.

- No other point of the cycle is self dual.

Proof. Since $\bar{S}^{\bar{w}}=\bar{S}$ is the unique greedy cycle of $\bar{S}=S$, It must be that $w=\bar{w}$. The rest then follows easily.

Problem 10 Describe the matching on self-dual secure states which results. 


\section{References}

[1] Gordon, Basil. Tilings of lattice points in Euclidean n-space. Discrete Math. 29 (1980), no. 2, 169_ 174.

[2] Meyerowitz, Aaron D. Tilings in $\mathbb{Z}$ with triples. J. Combin. Theory Ser. A 48 (1988), no. 2, 229-235. 